\title{
Multiple strokes and cerebral microangiopathy in a patient with Churg-Strauss syndrome
}

\author{
Vitalie Văcăraș ${ }^{1,2}$, Roxana-Maria Radu ${ }^{1,2}$, Enia Cucu ${ }^{1,2}$, \\ Fior Dafin Mureșanu ${ }^{1}$
}

1) Department of Neurology Science, University of Medicine and Pharmacy Cluj-Napoca, Romania

2) Department of Neurology, County Emergency Hospital Cluj, ClujNapoca, Romania

DOI: $10.15386 / \mathrm{mpr}-1587$

Manuscript received: 31.01 .2020

Received in revised form: 13.07.2020

Accepted: 25.11.2020

Address for correspondence:

roxana.radu2493@gmail.com

This work is licensed under a Creative Commons Attribution-NonCommercialNoDerivatives 4.0 International License

\begin{abstract}
Eosinophilic granulomatosis with polyangiitis (EGPA) is a multisystemic disease that mainly affects the lungs and skin. It is considered to be a small and mediumvessel vasculitis. Although neurologic manifestations of EGPA are reported, usually consisting of peripheral neuropathy, central nervous system manifestations are quite rare, those described being cerebral infarctions or hemorrhages.

We present the case of a 79-year-old woman diagnosed in 2016 with EGPA, being treated with Prednisone and Azathioprine, who presented to the Neurological Emergency Department with right hemiplegia, dysmetria in the left arm and right hemi-hypoesthesia. CT (computed tomography) and MRI (Magnetic resonance imaging) findings on admission described lacunar strokes. The patient presented with low creatinine clearance on admission (positive for chronic renal disease), grade III hypertension, ischemic cardiomyopathy and right calf deep vein thrombosis. The patient was started on neuroprotective and neurotrophic treatment associated with parenteral hydration, anticoagulant and hypotensive drugs. The patient's symptoms partially improved, with possibility of independently maintaining a sitting position and upright stance with unilateral sustenance at discharge.
\end{abstract}

Patients suffering from vasculitides must be carefully observed in order to prevent or treat complications that may emerge.

Keywords: cerebral microangiopathy, multiple strokes, chronic renal disease, Churg-Strauss syndrome

\section{Introduction}

Eosinophilic granulomatosis with polyangiitis (EGPA), formerly known as Churg-Strauss syndrome is a necrotizing small to medium-vessel vasculitis $[1,2]$. It is considered to be one of the three ANCA-associated vasculitides that include granulomatosis with polyangiitis and microscopic polyangiitis. It also has a higher rate of nervous system involvement compared to the other two vasculitides [3].

EGPA mainly presents with respiratory symptoms, such as asthma, but can extend to involving any organ [3]. Nervous system involvement usually consists of peripheral nerve symptoms such as multiplex mononeuropathy (with a frequency of $55-75 \%$ ) [4]. Central nervous system (CNS) is less commonly involved, with rates varying between different studies (6-39\% frequency) [4].

\section{Case report}

We present the case of a 79-yearold woman who was transferred to the Neurological Emergency Department after suffering a fall without loss of consciousness due to sudden onset of right hemiplegia. The patient had a known history of multiple ischemic strokes, grade III hypertension, ischemic cardiomyopathy and stage 3 chronic renal disease.

The patient was diagnosed in 
2016 with Churg Strauss Syndrome after multiple blood works that showed hypereosinophilia, high rheumatoid factor titer, elevated inflammatory markers and positive perinuclear anti-neutrophil cytoplasmic antibodies (P-ANCA). She was treated with prednisone $10 \mathrm{mg} / \mathrm{day}$ at first and then continued with azathioprine $50 \mathrm{mg}$ twice daily.

At the first clinical evaluation the patient was bed bound, slightly disoriented and presented a motor deficit with $1 / 5$ muscular strength grade in the lower right extremity and $3 / 5$ in the upper extremity, Babinski sign on the right, dysmetria with hypermetria in the left arm, right hemihypoesthesia and painful paresthesia of the lower limbs. She had dehydrated skin and mucosae, Bouchard and Heberdeen nodules on both hands, no pathological respiratory sounds, $\mathrm{SaO} 2$ of $98 \%$, BP of $150 / 90 \mathrm{mmHg}$, $80 \mathrm{bpm}$, rhythmic cardiac activity, holosystolic murmur all over the precordium and reduced pulses in both lower extremities. Her right calf was larger in diameter which led us to suspect deep venous thrombosis. A urinary catheter was installed on the first day after admission as the patient presented difficulty in urinary emission. An emergency cerebral CT was done, which showed an ischemic area in the profound region of the left sylvian artery and multiple infracentimetric hypodense lesions of bilateral periventricular locations (Figure 1).

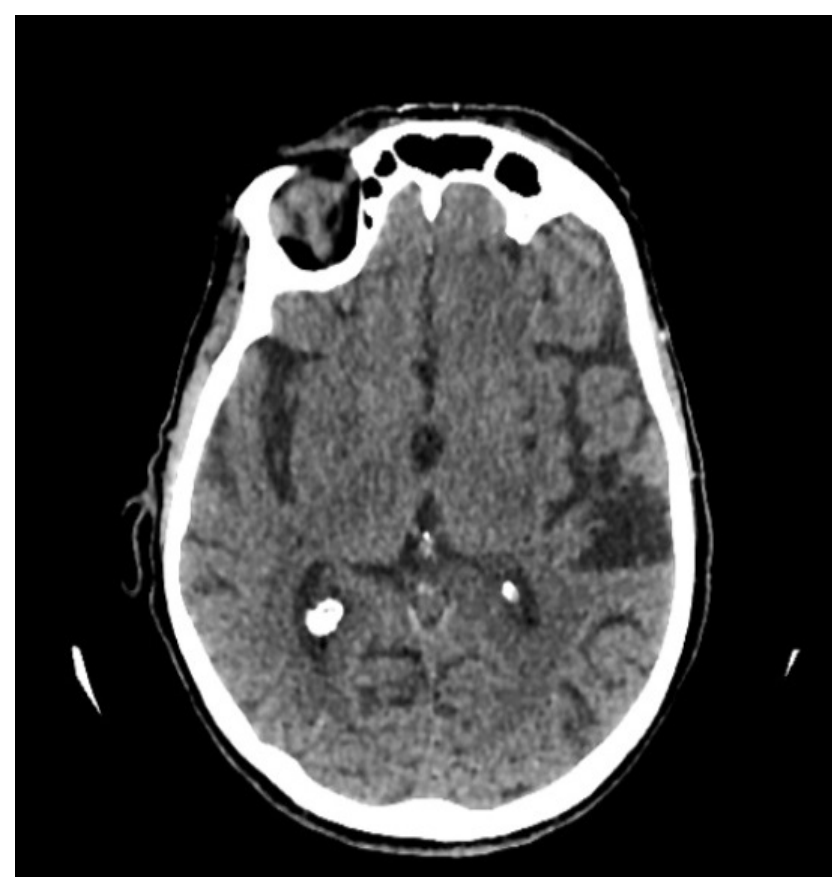

Figure 1. Cerebral CT (axial section) showing multiple infracentimetric hypodense lesions.

Blood workup on admission showed high creatinine levels $(3.74 \mathrm{ml} / \mathrm{dl})$ and urea levels $(177 \mathrm{ml} / \mathrm{dl})$ with a creatinine clearance of $10 \mathrm{ml} / \mathrm{min} ; 24$ hours proteinuria presented with a value of $1603 \mathrm{mg} / 24 \mathrm{~h}$. White blood cell count (WBC) showed decreased leucocytes $(2.96$ 10^9/L) with hypereosinophilia $(7.3 \%)$ and normochromic normocytic anemia ( $\mathrm{Hb}$ of $8.4 \mathrm{~g} / \mathrm{dl})$.

Nephrology consult recommended parenteral treatment with $\mathrm{NaCl} \quad 0.9 \% \quad 1500 \mathrm{ml} /$ day, furosemide treatment in case of hyperhydration, monitoring creatinine clearance and possibly starting erythropoietin and iron treatment in the future.

During hospital stay, the patient creatinine clearance slowly improved, with a value of $25 \mathrm{ml} / \mathrm{min}$ at discharge. Hemoglobin levels varied, with lowest value being at $7.2 \mathrm{mg} / \mathrm{dl}$, but transfusion was not required. A blood panel was ordered which showed anisocytosis with macrocytes and normocytes.

Inflammatory markers were high, with an erythrocyte sedimentation rate (ESR) of $55 \mathrm{~mm} / \mathrm{h}$ and a CRP of $7.36 \mathrm{mg} / \mathrm{dl}$ at admission. No infection was discovered and at discharge the CRP value decreased to $1.70 \mathrm{mg} / \mathrm{dl}$, but ESR remained elevated at $51 \mathrm{~mm} / \mathrm{h}$ (most probably due to the patient underlying rheumatologic pathology).

Venous Doppler evaluation was performed which confirmed the diagnosis of deep vein thrombosis of the right calf. The patient was started on Fraxiparine 3800 UI $0.4 \mathrm{ml}$ associated with Acenocumarol $2 \mathrm{mg}$ with INR monitoring. Due to the limitation imposed by the creatinine clearance, the anticoagulant doses could not be elevated and INR value failed to reach the desired value of between $2-3$, so the patient was switched to NOAC (New Oral Anticoagulants) (Apixaban $5 \mathrm{mg}$ twice a day). Blood analysis also showed increased pancreatic enzymes (amylase $=267 \mathrm{mg} / \mathrm{dl}$ and lipase $550 \mathrm{mg} / \mathrm{dl}$ ). An abdominal echography was ordered which proposed the suspicion of cholecystitis with pancreatic reactivity. A surgical consult was undergone which recommended an abdominal $\mathrm{CT}$. The imaging examination showed a cholecyst with inhomogeneous content, most probably biliary sludge with no other pathological modifications. Over the course of hospitalization the enzyme levels returned within normal values.

Due to its superiority in detecting lesions caused by vasculitic pathology, a cerebral MRI was performed. It showed large areas of T2 hypersignal located in the white substance, mostly periventricular, in the semioval centers and subcortically in both hemispheres. In the right parietal lobe a $15 / 12 \mathrm{~mm}$ T2 hyperintense lesion was described without diffusion restriction, most probably the result of an old ischemic stroke. On the SWI (Susceptibility weighted imaging) sequence multiple millimetric hypointense lesions were found and described as hypertensive microangiopathy (Figure 2). 

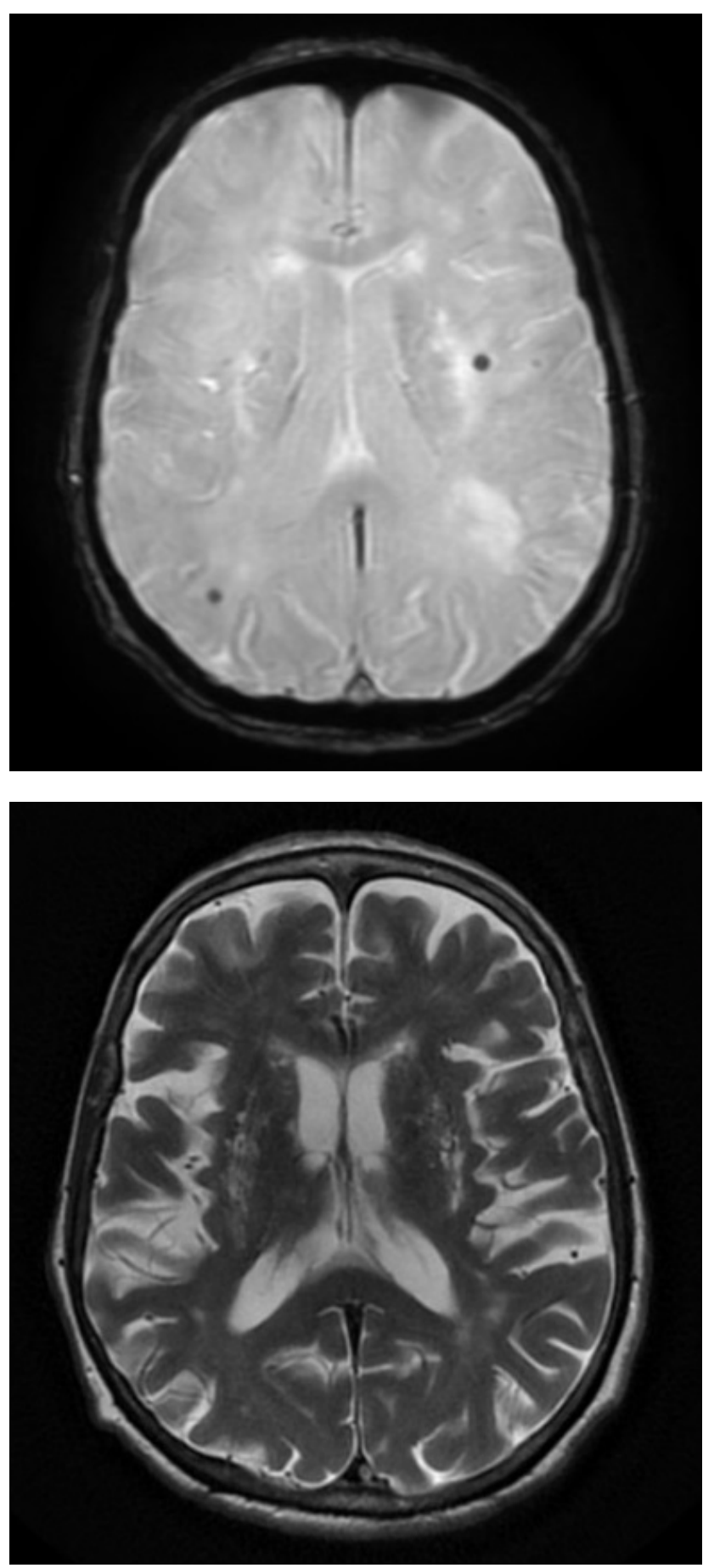

Figure 2. Cerebral MRI - SWI (first image) and T2 (second image) shows multiple microangiopathic lesions.

In conclusion, the examination established the diagnosis of supratentorial leukoaraiosis, multiple lacunar strokes in the fronto-parietal carotid vascular regions and old right parietal and left frontal ischemic strokes.

A psychological examination was conducted, which proved a moderate cognitive dysfunction with a MMSE (Mini-Mental State Exam) of 20/30, memory deficiency and fluctuating emotional reactivity.

The patient's treatment was limited because of the low value of the creatinine clearance. It consisted of a neuroprotective and neurotrophic agent (Cerebrolysin $215.2 \mathrm{mg} / \mathrm{ml}$ ). Hydration was provided with intravenous sodium chloride $0.9 \% 500 \mathrm{ml} ; 10 \%$ glucose $500 \mathrm{ml}$ daily was associated for nutritional purpose, as it was presumed safe to use, given the small dimension of stroke. Oral and subcutaneous anticoagulants, PPI (proton pump inhibitors), calcium channel blocker (amlodipine $10 \mathrm{mg}$ ) and beta blocker (carvedilol $6.25 \mathrm{mg}$ ) were also given. Compression stockings were complementary used in the treatment of the right calf vein thrombosis.

The patient's symptoms improved, with partial recovery of muscular strength, with possibility of independently maintaining a sitting position and upright stance with unilateral sustenance.

She was discharged from our department and guided to continue investigations in a Nephrology department with possibility of kidney biopsy for determining the cause of her chronic renal failure.

\section{Discussion}

When suspecting cerebral angiitis, imaging investigations play a crucial role in securing a diagnosis. MRI is the investigation of choice [5]. The differential diagnosis is impressively broad, consisting of pathologies such as coagulation disorders, embolic diseases and degenerative vascular pathologies. The two most common mimics of cerebral vasculitis are embolic strokes and intracranial atherosclerosis $[5,6]$.

In our case the diagnosis of EGPA was made three years prior based on immunological and biochemical investigations.

Churg Strauss syndrome is a rare systemic, necrotizing vasculitis affecting small-medium vessels.

Its clinical manifestations differentiate the disease into two subsets that are dominated either by vasculitis or by eosinophilic manifestations [7]. The disease course is divided into three phases: prodromal, eosinophilic and vascular phase [7].

The eosinophilic phase is dominated by lung, cardiac and gastrointestinal symptoms. Eosinophilic infiltrates tend to locate in the endo-myocardium, but may also affect the coronary arteries, the pericardium and the valves [1]. In our patient's case, ischemic cardiomyopathy with hypertension and holosystolic murmurs due to valvular defects were present. Additionally, patients present a higher risk of thrombo-embolic events, such as pulmonary embolism or deep vein thrombosis, the latter being diagnosed in our patient [1].

As for neurological involvement, peripheral neuropathy is a cardinal feature of the vasculitic phase, usually affecting between 46 to $75 \%$ of EGPA patients $[1,8]$. Nerves most frequently involved are common 
peroneal, internal popliteal, less frequently those of the arm, and lastly the cranial nerves [8]. Most common pattern is represented by mononeuritis multiplex (68\% of cases), that may later evolve into symmetric ( $28 \%$ of cases) or asymmetric ( $4 \%$ cases) polyneuropathies $[1,9]$. In our case, the patient described paresthesia and neuropathic pain located in both lower limbs.

Central nervous system involvment in EGPA is rare, with only $6-10 \%$ of all cases (and $25 \%$ of cases with peripheral nervous system involvement) being described. Intracranial vasculitis result in cerebral infarctions or hemorrhage [7,9]. T2-weighted MRI signal is more specific in detecting vasculitis derived lesions. Imaging findings vary, lesions being similar to those seen in other types of vasculitides, but mostly consist of macro or microinfarctions $[8,10]$. In our patient's case, the MRI conducted showed both multiple lacunar strokes and large ischemic strokes.

The kidney is another site affected by ANCA positive EGPA (one quarter of cases), with symptoms varying from isolated urinary abnormalities to rapidly progressive glomerulonephritis. There have been reports of patients that presented with chronic renal failure at diagnosis [7,11]. Our case presented with a creatinine clearance of $10 \mathrm{ml} /$ min at admission (stage $5 \mathrm{KDOQI}$ ), which proved to be a challenge in treating both the stroke, but also the vasculitis. Azathioprine dose was reduced from $100 \mathrm{mg}$ to $50 \mathrm{mg}$ a day to better suite the renal filtration. Kidney biopsy was recommended in order to decipher the patient's histological type of glomerulonephritis.

\section{Conclusion}

Churg-Strauss syndrome is a rare necrotizing, medium to small-vessel vasculitis with a higher rate of neurological symptoms compared to other ANCAassociated vasculitides. Central nervous system involvement is rare, but it may prove to be life threatening due to ischemic strokes or hemorrhages.

Although improvements have been made in treatment and prognosis, the disease remains a challenge to clinicians, due to its multiple pathological involvements. Rapid and correct diagnosis and treatment are mandatory in order to decrease the risk of complications.

As for our case, the diagnosis of Churg-Strauss had already been made, but it is important to observe that even with appropriate treatment the patient still developed life altering complications. The motor deficit that resulted due to the ischemic stroke will necessitate rehabilitation and kinetotherapy. Also, the implications involving the kidney disease represent an important impediment in correct treatment and dosage of drugs.

\section{References}

1. Vaglio A, Buzio C, Zwerina J. Eosinophilic granulomatosis with polyangiitis (Churg-Strauss): state of the art. Allergy. 2013;68:261-273.

2. Zuo N, Du Y, Liu N, Feng J, Wang L. Eosinophilic granulomatosis with polyangiitis and advanced renal failure in a case of glomerulosclerosis. Int J Clin Exp Pathol 2016;9:2477-2481

3. Oiwa H, Mokuda S, Matsubara T, Funaki M, Takeda I, Wamawaki $T$, et al. Neurological Complications in Eosinophilic Granulomatosis with Polyangiitis (EGPA): The Roles of History and Physical Examinations in the Diagnosis of EGPA. Intern Med. 2017;56:3003-3008.

4. Wolf J, Bergner R, Mutallib S, Buggle F, Grau AJ. Neurologic complications of Churg-Strauss syndrome - a prospective monocentric study. Eur J Neurol. 2010;17:582-588.

5. Berlit P. Diagnosis and treatment of cerebral vasculitis. Ther Adv Neurol Disord. 2010;3:29-42.

6. Byram K, Hajj-Ali RA, Calabrese L. CNS Vasculitis: an Approach to Differential Diagnosis and Management. Curr Rheumatol Rep. 2018;20:37.

7. Greco A, Rizzo MI, De Virgilio A, Gallo A, Fusconi M, Ruoppolo G. et al. Churg-Strauss syndrome. Autoimmun Rev. 2015;14:341-348.

8. Nguyen Y, Guillevin L. Eosinophilic Granulomatosis with Polyangiitis (Churg-Strauss). Semin Respir Crit Care Med. 2018;39:471-481.

9. Zhang S, Yuan D, Tan G. Neurological Involvement in Primary Systemic Vasculitis. Front Neurol. 2019;10:430.

10. Abdel Razek AA, Alvarez H, Bagg S, Refaat S, Castillo M. Imaging spectrum of CNS vasculitis. Radiographics. 2014;34:873-894.

11. Sinico RA, Di Toma L, Maggiore U, Tosoni C, Bottero $\mathrm{P}$, Sabadini E, et al. Renal involvement in Churg-Strauss syndrome. Am J Kidney Dis. 2006;47:770-779. 